\title{
Nurse home visits with or without alert buttons versus usual care in the frail elderly: a randomized controlled trial
}

\author{
Jesús Favela' \\ Luis A Castro ${ }^{2}$ \\ Francisco Franco-Marina ${ }^{3}$ \\ Sergio Sánchez-García ${ }^{4}$ \\ Teresa Juárez-Cedillo ${ }^{4}$ \\ Claudia Espinel Bermudez ${ }^{4}$ \\ Julia Mora-Altamirano ${ }^{4}$ \\ Marcela D Rodriguez ${ }^{5}$ \\ Carmen García-Peña ${ }^{4}$ \\ 'Center for Scientific Research \\ and Higher Education of Ensenada, \\ Ensenada, Baja California, Mexico; \\ ${ }^{2}$ Sonora Institute of Technology, \\ Ciudad Obregon, Mexico; ${ }^{3}$ National \\ Institute of Respiratory Diseases, \\ Mexican Ministry of Health, Mexico \\ City, Mexico; ${ }^{4}$ Epidemiologic and \\ Health Service Research Unit, Aging \\ Area, XXI Century National Medical \\ Center, Mexican Institute of Social \\ Security, Mexico City, Mexico; ${ }^{5}$ School \\ of Engineering, $\mathrm{MyDCl}$, Autonomous \\ University of Baja California, Mexicali, \\ Mexico
}

Correspondence: Carmen Garcia-Peña Unidad de Investigación Epidemiológica y en Servicios de Salud/Area de Envejecimiento, Edificio Corce, 3er piso, Centro Médico Nacional Siglo XXI,

Av Cuauhtémoc \#330, Col Doctores, Deleg Cuauhtémoc, 06725 México DF, México

Tel +525555 192724

Email mcgarciapena@gmail.com
This article was published in the following Dove Press journal:

Clinical Interventions in Aging

22 January 2013

Number of times this article has been viewed

Objective: To assess whether an intervention based on nurse home visits including alert buttons $(\mathrm{NV}+\mathrm{AB})$ is effective in reducing frailty compared to nurse home visits alone (NV-only) and usual care (control group) for older adults.

Design: Unblinded, randomized, controlled trial.

Setting: Insured population covered by the Mexican Social Security Institute living in the city of Ensenada, Baja California, Mexico.

Participants: Patients were aged over 60 years with a frailty index score higher than 0.14 . Intervention: After screening and informed consent, participants were allocated randomly to the control, NV+AB, or NV-only groups.

Measurements: The primary outcome was the frailty score 9 months later. Quality of life, depression, comorbidities, health status, and health service utilization were also considered.

Results: The framing sample included 819 patients. Of those, 591 were not located because they did not have a landline/telephone (341 patients), they had died (107), they were ill (50), or they were not currently living in the city (28). A screening interview was applied to 228 participants, and 57 had a score $\leq 0.14,171$ had $\geq 0.14$, and 16 refused to complete the baseline questionnaire. A home visit was scheduled for 155 patients. However, 22 did not complete the baseline questionnaire. The final 133 subjects were randomized into the NV+AB ( $n=45)$, NV-only $(n=44)$, and control $(n=44)$ groups. There were no statistically significant differences in the baseline characteristics of the groups. The mean age overall was 76.3 years (standard deviation 4.7 ) and $45 \%$ were men. At the baseline, $61.65 \%$ were classified as frail. At end of follow-up the adjusted prevalence of frailty in $\mathrm{NV}+\mathrm{AB}$ group was $23.3 \%$ versus $58.3 \%$ in the control group.

Conclusion: An intervention based on $\mathrm{NV}+\mathrm{AB}$ seems to have a positive effect on frailty scores.

Keywords: gerontechnology, frailty, elderly

\section{Introduction}

Population aging is becoming one of the most important demographic phenomena worldwide. It is the product of the clear decreases in birth and mortality rates and an increase in life expectancy, which are reflections of the socioeconomic progress of countries. ${ }^{1}$ The world population aged 60 years and older will grow from approximately 770 million in 2010 to an estimated 1 billion in 2020, and $20 \%$ of these people will be concentrated in developing countries. ${ }^{2}$

This demographic shift is happening twice as fast in Mexico than in many other countries, and both the absolute and relative numbers of older adults in the population are quickly increasing. ${ }^{3}$ The population of older Mexicans grew only $1.4 \%$ in the last 50 years (1950-2000) but will grow $17.7 \%$ in the next 50 years (2000-2050). 
In absolute terms, this trend means that in 2050 there will be 166.5 older adults for every 100 children. $^{4}$

As in most Latin American countries, Mexico is experiencing what is termed a "mixed" epidemiological transition, with increasing prevalence of chronic diseases and a marked decrease in communicable diseases in some regions of the country, while still suffering from moderate or high incidences of communicable diseases in others. According to Palloni et $\mathrm{al}^{1}{ }^{1}$ in addition to the fact that neither Mexico nor any other country in Latin America has the public or private institutional context to respond to the changing social and healthcare demands imposed by a growing elderly population, a highly compressed aging process will take place in the midst of weakening economies, changing intergenerational relations, and narrowing access to medical resources and healthcare.

This situation presents not only a demographic challenge, but also a social one because existing governmental support structures may be unable to meet this older population's health and social support needs. The unprecedented increase in the number of elderly subjects has uncovered the limited capacity of health systems to respond to their growing needs. Increasing needs coupled with the reduction of family support ${ }^{4-8}$ pose further challenges to the current unavailability of optimal resources. Given the lack of long-term health and social care strategies for the older population in Mexico, it is imperative that the health sector finds innovative strategies to cater to the needs of the growing older population.

Several programs based on nurse home visits, whether focused on one specific issue or with a more comprehensive perspective, have been reported in the literature. Evidence of their effectiveness is mixed. Some authors have reported no impact, ${ }^{9,10}$ whereas others have concluded that such programs have positive effects on mortality rates and functional decline. ${ }^{11}$ No single study has reported a combination of nurse home visits and the use of supportive technology. The existing evidence is encouraging enough to continue research in this area. We conducted a randomized clinical trial to assess whether an intervention based on nurse home visits plus alert buttons $(\mathrm{NV}+\mathrm{AB})$ is effective in terms of reducing and/or preventing further frailty in older adults compared with an intervention including nurse home visits alone (NV-only) and the usual care intervention (control group) for older adults insured by the Mexican Social Security Institute (IMSS).

\section{Methods}

\section{Design}

We performed a randomized, unblinded, controlled clinical trial. Three groups were assembled: one group received exclusively periodic nurse visits (NV-only group), another group received periodic nurse visits including an alert button (NV+AB group) and the third group received usual care (control group). The Ethics Committee at the IMSS approved the protocol (reference 2008-785021). Written consent was obtained from all subjects who agreed to participate in the study, and the study complied with the principles of the Declaration of Helsinki and its recommendations.

\section{Setting}

The participants were men and women with insurance supplied by the IMSS who lived in the city of Ensenada, Baja California and were registered with the Family Medicine Clinic. IMSS is a mandatory social security system that offers a comprehensive package of benefits, including healthcare at all levels, as well as economic benefits such as a retirement pension. IMSS-insured workers and their close relatives are affiliated with a Family Medicine Unit based on their home address. Elderly individuals insured by the IMSS are more likely than their non-IMSS affiliated counterparts to have 6 or less years of education.

The sample size calculation was based on a minimum expected frailty index difference of 0.04 between interventions based on NV-only and NV+AB versus usual care. According to the calculations, 45 participants were required with an alpha level of 0.05 and a beta level of 0.20 .

\section{Recruitment of participants}

The screening process was conducted via telephone interviews by three trained interviewers. In addition to location data and willingness to participate, relevant data for inclusion as inputs in the generation of a frailty index were obtained. ${ }^{12}$ Briefly, the frailty index integrates 34 variables following the scoring system suggested by Rockwood et al. ${ }^{13}$ Their approach to measuring frailty is to generate a simple count of different symptoms, illnesses, and other conditions referred to as deficits that an individual presents and use this value to conclude a certain frailty status. ${ }^{14}$ Most of the variables indicate the presence or absence of a particular deficit. A score of 1 was given for the presence of frailty and a score of 0 otherwise. The frailty index was thus defined as the proportion of the total number of deficits that an individual has with respect to the 34 deficits included. The data were simultaneously captured in Epidata ${ }^{15}$ and programmed to obtain the frailty index score. Interviewers verified the values obtained, and those participants with a score equal to or higher than 0.14 were invited to participate.

\section{Baseline and final data collection}

Baseline interviews were conducted at the participants' homes between February and March 2009. Trained interviewers not 
involved in the intervention performed the baseline and the final data collections. The information collected included sociodemographic data, prescribed medications, health services utilization, and self-report of comorbidity, cognitive impairment, depression, social support, quality of life, activities of daily living (ADL), nutritional status, and frailty, which were assessed with the following instruments.

\section{Comorbidity}

Comorbidity was evaluated using the Charlson Index. ${ }^{16}$

Participants were asked whether they had a diagnosis of 19 chronic diseases according to the World Health Organization's International Classification of Diseases (ICD-10). ${ }^{17}$ Each disease was scored from 1 to 6 according the risk of death. Severity of comorbidity was established under a total score as follows: $1=$ not severe, $2=$ mild, $3=$ moderate, and $4=$ severe.

\section{Cognitive function}

Cognitive function was assessed using a validated Spanish version $^{18,19}$ of the Folstein ${ }^{20}$ Mini-Mental State Examination (MMSE). The MMSE evaluates memory, orientation to space and time, calculation, language, and word recognition, with scores that range from 0 to 30 points with lower scores indicating poorer cognitive ability. Cognitive impairment was determined using a cutoff point of $\leq 23$, adjusted by age.

\section{Depression}

Depression was measured using a Spanish version ${ }^{21}$ of the Center for Epidemiological Studies Depression Scales (CES-D), ${ }^{22}$ which was developed to measure levels of depressive symptoms in population studies, as well as to identify probable clinical depression. The original scale includes 20 items that inquire about mood, somatic complaints, interaction with others and motor functioning during the week preceding the interview. Each of the responses in this version includes a Likert scale of 4 points, with a range of $0-3$, and the categories of responses in terms of days per week, from less than 1 day to 5 or 7 days. To obtain the final score, these responses are summed and a scale of 0-60 is obtained, where the higher scores indicate greater impairment and the cutoff point to indicate depression is set at 16. In the Spanish version, the CES-D scale has shown high internal consistency and adequate validity. ${ }^{21}$

\section{Quality of life}

The evaluation of this variable was made based on the concept of health-related quality of life (HRQoL) of elderly adults. ${ }^{23}$
In this study, we used the Medical Outcomes Study 36-item Short-Form Health Survey (SF-36). ${ }^{24}$ The Spanish version has been validated in a Mexican population. ${ }^{25}$ The SF-36 consists of 36 items that measure HRQoL during the 4 weeks prior to the administration of the questionnaire through the following eight scales or dimensions: physical function (PF), role limitations due to physical health problems (RP), bodily pain (BP), social functioning (SF), general mental health that includes psychological stress $(\mathrm{MH})$, role limitations due to emotional problems (RE), vitality, and energy or fatigue (VT). Scoring was performed using the RAND algorithm, ${ }^{26}$ which produces an overall SF-36 score as well as individual scores for the eight subscales. Scores range from 0 to 100 , with higher scores indicating better HRQoL.

\section{ADL}

Disability related to basic ADL and instrumental activities of daily living (IADL) was used to identify disability in the participants. For ADLs, ${ }^{27}$ subjects were asked whether they needed help for bathing, dressing, toileting, transferring, and feeding. Incontinence was also evaluated. For IADLs ${ }^{28}$ participants reported their ability to perform eight IADLs, which were adjusted by sex: using the telephone, shopping, grooming, housekeeping, doing laundry, using transportation, handling medications, and handling finances. For each domain of disability, if the participants indicated that they were unable to perform at least one of the activities without assistance, they were considered as having an IADL or ADL disability.

\section{Nutritional status}

Height and weight were reported by the participants, and body mass index (BMI) was calculated. ${ }^{29}$ The short version of the Mini Nutritional Assessment (MNA) was applied. ${ }^{30}$ This is a validated nutritional screening and assessment tool that can identify geriatric patients age 65 years and above who are malnourished or at risk of malnutrition.

\section{Frailty}

In addition to the measures of frailty collected in the screening phase, the frailty criteria developed by Fried et $\mathrm{al}^{31}$ were also included using the five following variables: unintended weight loss, exhaustion, low physical activity, slowness, and weakness. Weight loss was defined as a selfreport of an unintentional loss of $4.5 \mathrm{~kg}$ or more in the prior year or after 9 months of follow-up. Exhaustion was identified by the self-report of two questions from the CES-D scale: ${ }^{21}$ "I felt that everything I did was an effort" and "I could not 
get going." Subjects answering a moderate amount of time or most of the time were categorized as frail in this dimension. Low physical activity level was measured by the International Physical Activity Questionnaire (IPAQ) ${ }^{32,33}$ Participants were identified as positive if their physical activity was scored as less than moderate. The pattern of activity was classified as "moderate" if it met either of the following criteria: three or more days of vigorous-intensity activity of at least 20 minutes per day or five or more days of moderate-intensity activity and/or walking of at least 30 minutes per day.

Slowness was identified if participants could not walk 8 feet or required longer than approximately 7 seconds to walk this distance. Participants were requested to walk straight and time taken was recorded using a chronometer.

Weakness was measured by grip strength and was adjusted by sex, with a cutoff for women of $17 \mathrm{~kg}$ and for men of $30 \mathrm{~kg}$.

Frailty was considered present if three or more of the former criteria were positive.

\section{Randomization}

A simple randomization process without blocking was designed using a computer-generated list. After informed consent was obtained by the interviewer, the supervisor called the research coordinator's office and the research assistant notified to which group the participant should be allocated.

\section{Training}

Nurses participating in both interventions (NV-only and $\mathrm{NV}+\mathrm{AB}$ ) were first given training about aging, geriatric syndromes, elements of pharmacology, the nurse-patient relationship, conducting personal interviews, health behavior change models, the process of negotiation and ethical aspects of home visits. In addition, the nurses participating in the $\mathrm{NV}+\mathrm{AB}$ group were trained in geriatric emergencies and the correct application of telephone triage protocols. They were also trained in the use of the alert button and cellular phone applications for triage protocols. ${ }^{34,35}$ Quality control was provided through regular patient visits, reviews of clinical files, and random telephone calls made by a medical supervisor.

\section{Interventions}

\section{Nurse home visits including alert button ( $\mathrm{NV}+\mathrm{AB})$}

Seven nurses with bachelor's degrees were each assigned between six and seven participants. Participants in the intervention group received weekly visits from a nurse over a period of 9 months. During the first visit, nurses performed a medical history and identified, together with the patient, areas of potential improvement, including home environmental modifications. Close relatives or the primary family caregivers were involved whenever possible. During visits, nurses and patients reviewed information from the baseline health questionnaire and discussed possible lifestyle changes. The nurses tried to guide their patients and their relatives towards a better health status by suggesting alternative methods of achieving the desired changes and negotiating specific targets. The nurses also reviewed the subjects' pharmacological treatments and adherence was encouraged. The patients led the process of negotiation. This group of patients was also able to contact their nurses on whenever they felt the need by pressing the alert button. The nurses immediately answered the call using a cellular phone, and used the triage protocols application installed on an iPod Touch (Apple, Cupertino, CA, USA) to resolve the phone call, including clinical emergencies.

\section{Nurse home visits only (NV-only)}

Four nurses with bachelor's degrees were each assigned between ten and eleven patients. The content of the intervention was the same as the intervention above described, but this group did not include emergency care or technological support via the alert button.

Grieving and farewell processes were discussed with the patients 1 month before intervention ended in both groups.

\section{Control group}

The control group received no intervention beyond the usual care at the Family Medicine Clinic.

All patients from all groups continued to receive usual care from their family physicians at the clinic.

\section{Outcome measures \\ Outcome}

The primary outcome was the change in the frailty phenotype measured by the Fried criteria ${ }^{31}$ between the screening and the final phase 9 months later.

\section{Statistical analysis}

All analyses were done with STATA software (v 12; Stata Corp, College Station, TX, USA). ${ }^{36}$ Variables were summarized through means and proportions, according to their scale of measurement. Baseline characteristics distributed unevenly among the groups were identified through a descriptive analysis. ${ }^{37}$ 
The prevalence of frailty, as defined by Fried et al, ${ }^{31}$ at the end of follow-up observed in the NV-only and the $\mathrm{NV}+\mathrm{AB}$ groups was compared with that observed in the control group. Since, possibly because of the relatively small sample size, several baseline characteristics were unevenly distributed among the groups, the prevalences of frailty were contrasted in a multiple logistic regression model, adjusting for baseline characteristics. ${ }^{38}$ Comparisons between each intervention group and the control group were tested using the likelihood ratio test and a $P$-value $<0.05$ indicated statistical significance.

Since there were deaths and losses to follow-up, we also present analysis considering these participants. In them, we assumed that at the end of follow up those who died would have been frail. For those lost to follow-up we imputed (using the "impute" procedure available in STATA) their final frailty status, based on their baseline levels in the following variables: frailty status, sex, marital status, years of school, IADL, cognitive impairment, depression, BMI category, and score on the PF and SF SF-36 subscales.

In addition, we also identified which participants changed their frailty status at follow-up, and present a descriptive analysis of these changes in the compared groups.

\section{Results}

The framing sample of the study was composed of 819 patients. Of these, 591 were not located for various reasons. Many did not have a landline telephone (341 cases), some had died (107 cases), some were not in a condition to complete the interview (50 cases), and others were not currently living in the city (28 cases) (Figure 1).

A telephone screening interview was administered to the remaining 228 participants. Using the established cutoff point of a frailty index score equal to or higher than 0.14 , of the 228 patients, 57 were excluded from the sample because they had a score lower than $0.14,171$ had a score equal to or higher than 0.14 , and 16 refused to complete the baseline questionnaire. Therefore, 155 patients were scheduled for home visits; however, 22 did not complete the baseline questionnaire. The final sample included 133 patients that were randomized into three groups: 45 patients were assigned to the $\mathrm{NV}+\mathrm{AB}$ group, 44 to the NV-only group, and 44 patients were assigned to the control group.

The overall follow up rate was $86.5 \%$ with five randomized participants dying during the follow-up (3.8\%) and 13 being lost to follow-up (9.7\%). The NV-only group lost $46.1 \%$ of participants to follow-up, but only $20 \%$ due to death, with a follow-up rate of $84.1 \%$, compared to $86.7 \%$ in the $\mathrm{NV}+\mathrm{AB}$ group and $88.6 \%$ in the control group. The $\mathrm{NV}$-only and $\mathrm{NV}+\mathrm{AB}$ groups had more similar proportions of deaths and losses to follow-up.

\section{Baseline characteristics}

The three compared groups had an average baseline age between 75 and 76 years and a similar age-group distribution (Table 1). Forty percent of participants in the NV+AB group were men, compared to $47.7 \%$ in the other two groups. The NV+AB group also had a lower proportion of participants living with a couple than the NV-only and control groups. Participants in this study had on average 5.4 years of education (standard deviation $=4.6$ years), but the NV-only group had a lower proportion of persons with nine or more years of education $(9.1 \%)$, and therefore less years of education on average, compared to the $\mathrm{NV}+\mathrm{AB}$ and control groups ( $20.0 \%$ and $22.7 \%$, respectively). Health care utilization during the previous 6 months was similar in the three compared groups and close to $80 \%$. Most participants in the study were able to perform ADL with percentages ranging between $73.3 \%$ and $81.8 \%$ in the NV+AB and control groups, respectively. Regarding instrumental activities, being able to walk in the street was reported with lower percentages, ranging between $56 \%$ to $52 \%$ in the NV-only and control groups, respectively.

Regarding clinical measures at baseline, participants in the study reported an average of 1.9 of the chronic diseases queried for calculating the Charlson index of comorbidity, with only $19.6 \%$ of them not reporting any of these diseases. Participants with a Charlson index of comorbidity score of 7 or more were more prevalent in the NV-only group (50\%) than in the other two groups (corresponding percentages: $43.2 \%$ in the control group and $40 \%$ in the $\mathrm{NV}+\mathrm{AB}$ group). One third of participants in the $\mathrm{NV}+\mathrm{AB}$ were positive for depression on the CES-D scale and the same proportion had cognitive impairment, as assessed by the MMSE. Lower frequencies of these conditions were observed in the NV-only and control groups.

Table 1 also compares the baseline frailty criteria for the frailty phenotype ${ }^{31}$ The proportion of participants meeting Fried's criteria to be considered frail was similar in the three compared groups, close to $45 \%$. Roughly, one in five or six participants reported recent unintentional weight loss, with the NV+AB group showing the lowest proportion (15.6\%). Exhaustion was reported in $25.6 \%$ of participants, with $33 \%$ of participants in the $\mathrm{NV}+\mathrm{AB}$ group reporting exhaustion. Weakness was found in $54.1 \%$ of participants, but this percentage was lower in the control and NV-only groups. 


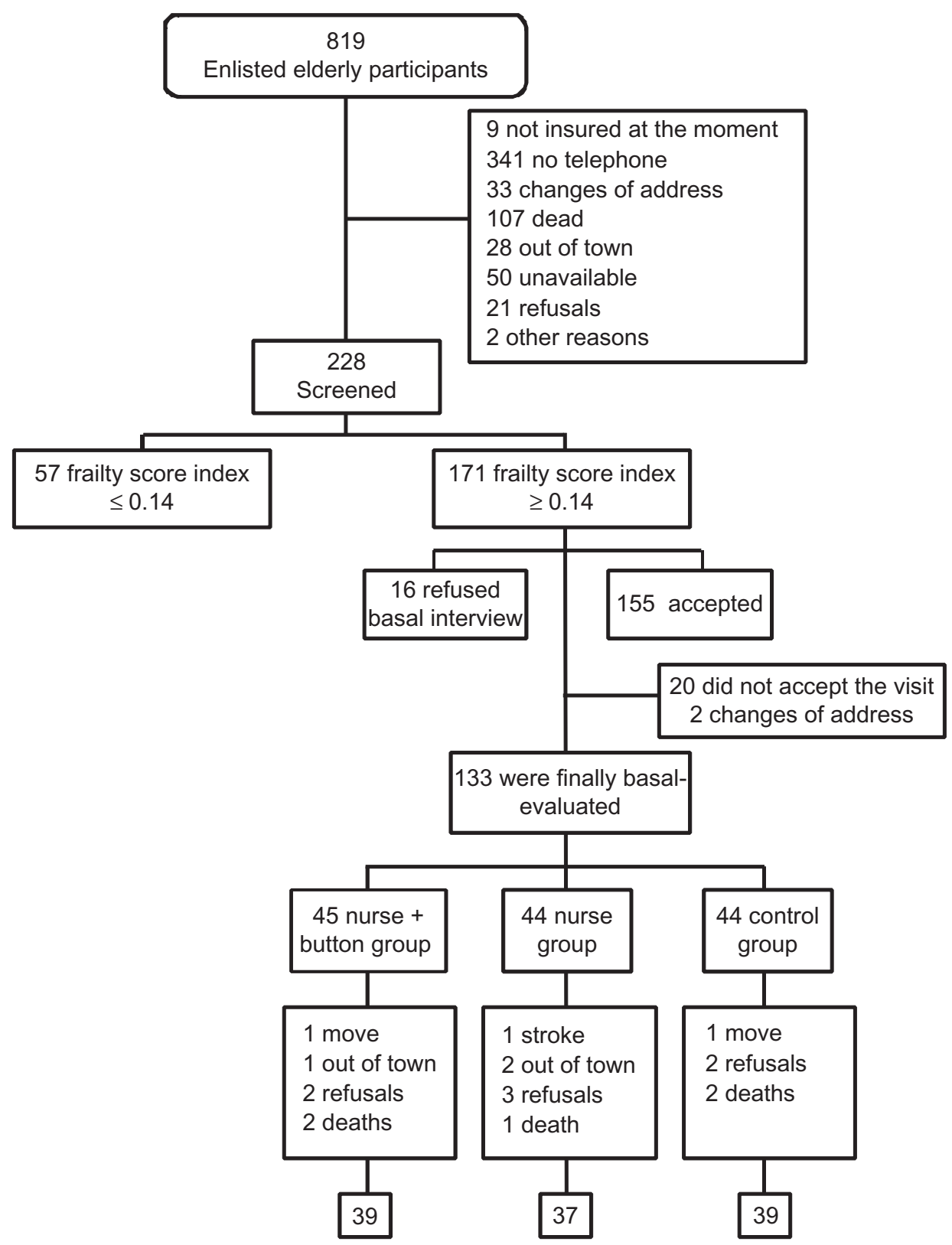

Figure I Flow chart of subjects throughout the study.

Practically all study participants had slow walking speed. In addition, $57.8 \%$ of participants in the $\mathrm{NV}+\mathrm{AB}$ group had a low physical activity level and for participants in the control group this level was much lower (43.2\%).

Table 2 presents the distribution of nutritional status indicators among the compared groups. Average weight showed a variation of $1.7 \mathrm{~kg}$ among the randomized groups whereas average height varied by $4 \mathrm{~cm}$. Underweight participants were more prevalent in the NV-only group (4.6\%), with the control and $\mathrm{NV}+\mathrm{AB}$ groups showing the same underweight prevalence $(2.3 \%)$. The average BMI in study participants was 27.4 , but in the $\mathrm{NV}+\mathrm{AB}$ group it was 28.7 with $31.8 \%$ of obese participants, compared to $23.3 \%$ and $20.5 \%$ in the control and NV-only groups, respectively. According to the MNA, 79.6\% of participants in the $\mathrm{NV}+\mathrm{AB}$ group had a normal nutritional status with only $2.3 \%$ malnourished, compared to $69.8 \%$ of participants in the control group with normal nutritional status and $7.0 \%$ with malnutrition.

General health perceptions on the SF-36 show the lowest proportion of participants in the $\mathrm{NV}+\mathrm{AB}$ group rating their current health as fair or poor and the lowest proportion of participants in the control group stating that their baseline health was worse than 1 year ago (Table 3 ). In addition, the scores for the quality of life dimensions measured by SF-36 
Table I Baseline sociodemographic and clinical characteristics among study groups

\begin{tabular}{|c|c|c|c|}
\hline & $\begin{array}{l}\text { Control } \\
(n=44) \\
(\%)\end{array}$ & $\begin{array}{l}\text { NV-only } \\
(n=44) \\
(\%)\end{array}$ & $\begin{array}{l}\text { NV+AB } \\
(n=45) \\
(\%)\end{array}$ \\
\hline \multicolumn{4}{|l|}{ Age-group } \\
\hline $70-74$ & 36.4 & 45.5 & 42.2 \\
\hline 75-79 & 34.1 & 34.1 & 37.8 \\
\hline $80-84$ & 22.7 & 15.9 & 13.3 \\
\hline $85-90$ & 6.8 & 4.6 & 6.7 \\
\hline \multicolumn{4}{|l|}{ Sex } \\
\hline Men & 47.7 & 47.7 & 40.0 \\
\hline \multicolumn{4}{|l|}{ Marital status } \\
\hline Living with a partner & 52.3 & 56.8 & 46.7 \\
\hline Widowed & 31.8 & 27.3 & 42.2 \\
\hline Living alone & 15.9 & 15.9 & 11.1 \\
\hline \multicolumn{4}{|l|}{ Years of school } \\
\hline $0-2$ & 20.5 & 34.1 & 26.7 \\
\hline $3-5$ & 34.1 & 20.5 & 26.7 \\
\hline $6-8$ & 22.7 & 36.4 & 26.7 \\
\hline $9+$ & 22.7 & 9.1 & 20.0 \\
\hline $\begin{array}{l}\text { Sought/received health services } \\
\text { in past } 6 \text { months (IMSS) }\end{array}$ & 79.6 & 84.1 & 82.2 \\
\hline $\begin{array}{l}\text { Total independence in activities } \\
\text { of daily living }\end{array}$ & 81.8 & 77.3 & 73.3 \\
\hline Able to walk in the street & 52.2 & 56.8 & 55.5 \\
\hline \multicolumn{4}{|l|}{ Charlson comorbidity index } \\
\hline $3-6$ & 56.8 & 50.0 & 60.0 \\
\hline $7-12$ & 43.2 & 50.0 & 40.0 \\
\hline Depression ${ }^{\mathrm{a}}$ & 22.7 & 29.6 & 33.3 \\
\hline Cognitive impairment ${ }^{\mathrm{b}}$ & 29.6 & 22.7 & 33.3 \\
\hline Frailtyc & 45.5 & 43.2 & 46.7 \\
\hline Unintentional weight loss & 20.5 & 18.2 & 15.6 \\
\hline Self-reported exhaustion & 18.2 & 25.0 & 33.3 \\
\hline Weakness & 52.3 & 50.0 & 60.0 \\
\hline Slow walking speed & 100.0 & 95.5 & 97.8 \\
\hline Low physical activity level & 43.2 & 52.3 & 57.8 \\
\hline
\end{tabular}

Notes: ${ }^{a} \mathrm{~A}$ score of 16 or more on the CES-D scale; ba score of 23 or less on MiniMental State Examination (MMSE); ' with frailty phenotype, according to Fried et al's criteria. ${ }^{31}$ Weight loss was defined as a self-report of an unintentional loss of $4.5 \mathrm{~kg}$ or more in the prior year or after 9 months of follow-up. Exhaustion was identified by self-report of two questions from the CES-D scale:21,22 "I felt that everything I did was an effort" and "I could not get going." Subjects answering "a moderate amount of time" or "most of the time" were categorized as frail in this dimension. Low physical activity level was measured by the International Physical Activity Questionnaire. ${ }^{32,33}$ Participants were identified as positive if their physical activity was scored as less than moderate. The pattern of activity classified as "moderate" is either of the following criteria: 3 or more days of vigorous-intensity activity of at least 20 minutes per day or 5 or more days of moderate-intensity activity and/or walking of at least 30 minutes per day. Slowness was identified if participants could not walk 8 feet or could not walk this distance in 7 seconds. Weakness was measured by grip strength adjusted by sex, with cutoffs for women of $17 \mathrm{~kg}$ and for men of $30 \mathrm{~kg}$.

Abbreviations: NV, nurse visits; $N V+A B$, nurse visits including an alert button CES-D, Center for Epidemiological Studies Depression; IMSS, Mexican Social Security Institute.

were lower for the $\mathrm{NV}+\mathrm{AB}$ group in all subscales, except in the PF subscale.

\section{Outcome evaluation}

The prevalence of frailty at the end of follow-up is shown in Figure 2. All prevalence figures were adjusted for baseline
Table 2 Baseline nutritional status among study groups

\begin{tabular}{llll}
\hline & $\begin{array}{l}\text { Control } \\
(\mathbf{n}=\mathbf{4 4})\end{array}$ & $\begin{array}{l}\text { NV-only } \\
(\mathbf{n}=\mathbf{4 4})\end{array}$ & $\begin{array}{l}\text { NV+AB } \\
(\mathbf{n}=\mathbf{4 5})\end{array}$ \\
\hline Weight, kg (mean [SD]) & $70.5(14.8)$ & $68.9(12.8)$ & $70.6(12.6)$ \\
Height, m (mean [SD]) & $\mathrm{I} .62(0.10)$ & $1.60(0.10)$ & $1.58(0.10)$ \\
WHO BMI category, ${ }^{29} \%$ & & & \\
Underweight & 2.3 & 4.6 & 2.3 \\
Normal & 39.5 & 27.3 & 22.7 \\
Overweight & 34.9 & 47.7 & 43.2 \\
Obese & 23.3 & 20.5 & 31.8 \\
Mini Nutritional Assessment (MNA), & \% & \\
Normal nutritional status & 69.8 & 75.0 & 79.6 \\
At risk of malnutrition & 23.3 & 20.5 & 18.2 \\
Malnourished & 7.0 & 4.6 & 2.3 \\
\hline
\end{tabular}

Abbreviations: BMI, body mass index; SD, standard deviation; WHO, World Health Organization; NV, nurse visits; NV+AB, nurse visits including an alert button.

variables that were unevenly distributed among the compared groups and related to frailty. Among those followed up, in the $\mathrm{NV}+\mathrm{AB}$ group the prevalence of frailty was $40 \%$ that found in the control group (adjusted prevalence of frailty $23.3 \% \mathrm{vs}$ $58.3 \%$, respectively). This result was statistically significant $(P<0.05)$. Similar results are obtained if deaths and losses to follow-up are included, assuming that all participants who died were frail and imputing the frailty status based on baseline variables in those lost to follow-up. Under this scenario, the $\mathrm{NV}+\mathrm{AB}$ group would have a $45 \%$ lower prevalence of frailty than the control group (adjusted prevalence of frailty $29.6 \% \mathrm{vs}$ $66.5 \%$, respectively). In addition, the NV-only group had a similar prevalence of frailty to the control group.

Table 3 Baseline SF-36 quality of life dimensions among study groups

\begin{tabular}{|c|c|c|c|}
\hline & $\begin{array}{l}\text { Control } \\
(n=44)\end{array}$ & $\begin{array}{l}\text { NV-only } \\
(n=44)\end{array}$ & $\begin{array}{l}N V+A B \\
(n=45)\end{array}$ \\
\hline \multicolumn{4}{|c|}{ In general, would you say your health is (\%) } \\
\hline Excellent/very good & 18.2 & 11.4 & 17.8 \\
\hline Good & 36.4 & 34.1 & 42.2 \\
\hline Fair/poor & 45.5 & 54.6 & 40.0 \\
\hline \multicolumn{4}{|c|}{ Compared to I year ago, how would } \\
\hline \multicolumn{4}{|c|}{ you rate your health in general now? (\%) } \\
\hline Better now than I year ago & 27.3 & 22.7 & 31.1 \\
\hline About the same as I year ago & 52.3 & 45.5 & 35.6 \\
\hline Worse now than I year ago & 20.5 & 31.8 & 33.3 \\
\hline \multicolumn{4}{|c|}{ SF-36 subscale (mean [SD]) } \\
\hline Physical functioning & $54.7(31.5)$ & $54.3(27.3)$ & $54.7(28.7)$ \\
\hline Role physical & $47.7(42.4)$ & $43.8(44.8)$ & $4 I . I(4 I .3)$ \\
\hline Bodily pain & $64.6(27.8)$ & $65.3(28.7)$ & $61.4(26.7)$ \\
\hline Vitality & $70.9(23.8)$ & $62.6(23.1)$ & $60.3(26.2)$ \\
\hline Social functioning & $79.3(27.0)$ & $76.7(26.5)$ & $71.9(33.2)$ \\
\hline Role emotional & $75.8(39.6)$ & $71.2(36.4)$ & $67.4(44.1)$ \\
\hline Mental health & $81.9(21.4)$ & $75.2(23.8)$ & $71.0(27.5)$ \\
\hline
\end{tabular}

Abbreviations: $N V$, nurse visits; $N V+A B$, nurse visits including an alert button; SF-36, 36-item Short-Form Health Survey; SD, standard deviation. 


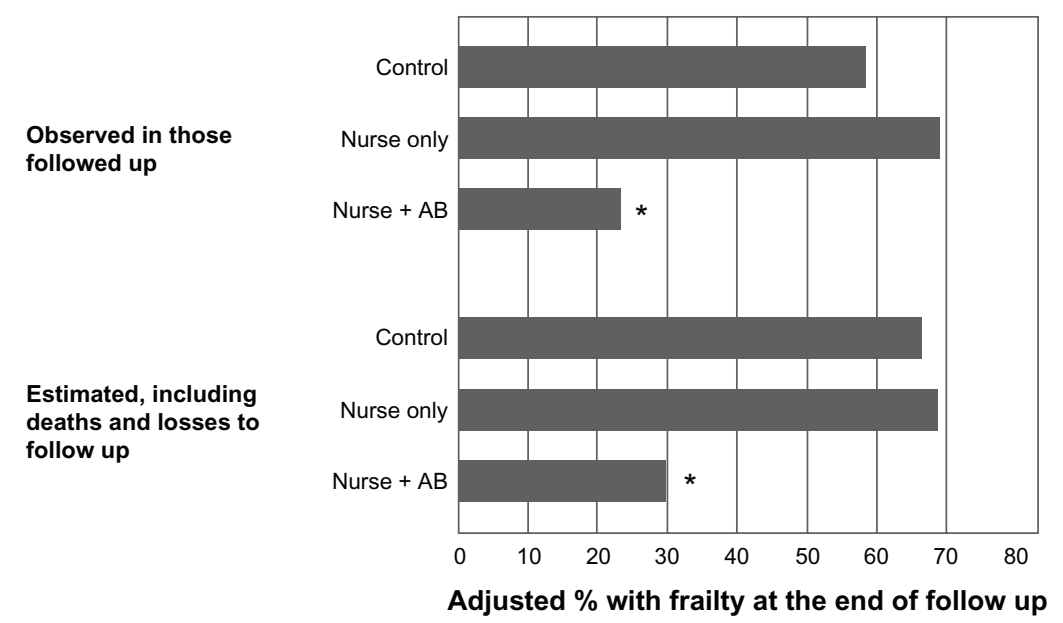

Figure 2 Percentages of participants with frailty at the end of follow-up in the three study groups.

Notes: Percentages presented are adjusted for baseline frailty, sex, marital status, years of school, independence in activities of daily living, cognitive impairment, depression, body mass index category, and score on the role physical and social functioning SF-36 subscales, through logistic regression. Adjusted percentages correspond to the expected frailty proportion of an average participant, in terms of the adjustment variables. $* P<0.05$, comparing the $N V+A B$ to the control group

Abbreviations: NV+AB, nurse visits including an alert button; SF-36, 36-item Short-Form Health Survey.

Finally, Figure 3 presents the changes in frailty and its components, occurring between the start and the end of follow up. Overall, $11.3 \%$ of participants were no longer frail at the end of follow-up, with the NV+AB group showing a slightly higher percentage of improvement than the other two groups $(12.8 \%)$. On the other hand, the $\mathrm{NV}+\mathrm{AB}$ group presented a considerably lower percentage of participants who developed frailty during the follow up (5.1\%) than those in the control or the NV-only group ( $12.8 \%$ and $24.3 \%$, respectively). In addition, the $\mathrm{NV}+\mathrm{AB}$ group showed the highest proportion of participants who were no longer exhausted and who improved their walking speed at the end of follow-up. In contrast, the NV-only group had the highest proportion of participants who became frail during the follow up (24.3\%), as well as the highest percentage of participants who developed exhaustion and the lowest percentages of participants who improved in their walking speed and their physical activity level.

\section{Discussion}

The most important findings of this study reveal that an intervention based on $\mathrm{NV}+\mathrm{AB}$ and mobile phones had a positive effect on the reduction of frailty, after adjusting for a number of confounders. However, positive results were also reported in the control group.

The current state of affairs in population aging in México and in other Latin American countries set the stage
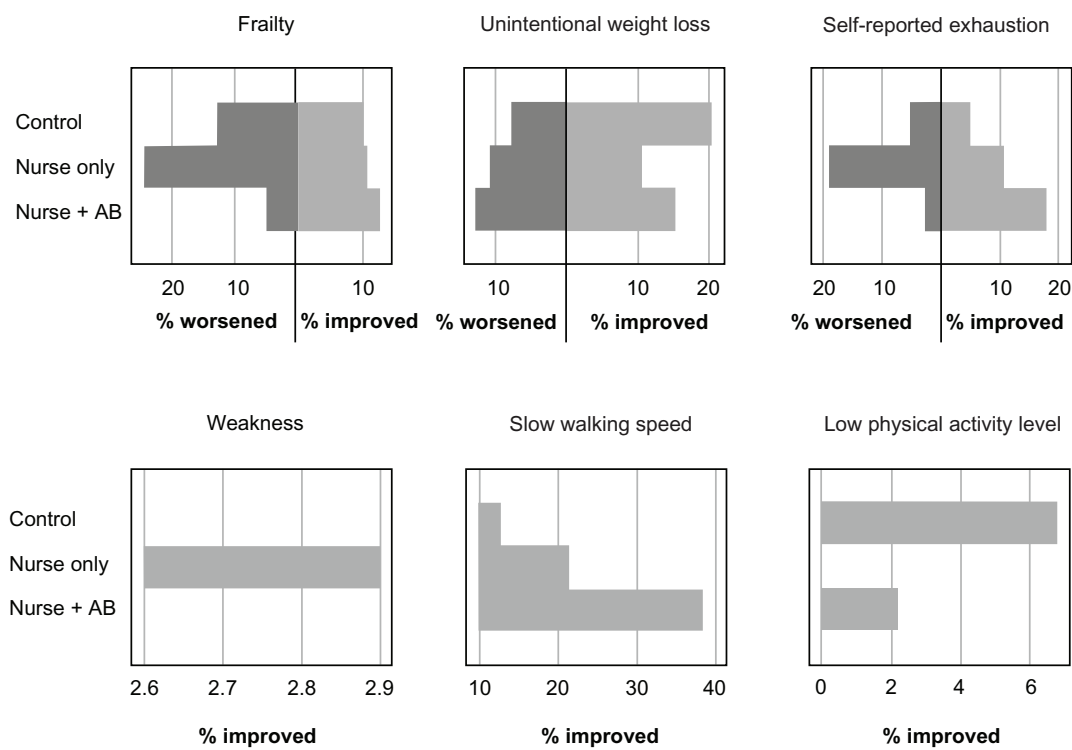

Low physical activity level

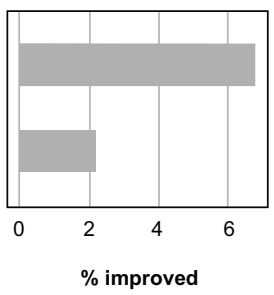

Figure 3 Percentages of participants who either improved or worsened in their frailty status or Fried's frailty criteria, ${ }^{31}$ during their follow-up. Abbreviation: $A B$, alert button. 
for this study. While an increasing cohort of the population is moving into the latter phases of life, the resources to fund both acute and long-term care are scarce. A recent report of the Organization for Economic Cooperation and Development ${ }^{39}$ reported that Mexico spends only $0.01 \%$ of its GDP on long-term care, whereas the average for the rest of the organization's members is approximately $1.5 \%$.

The health system is not prepared to face such a challenge. Hospitals and primary care clinics report growing rates of utilization and high probabilities of acute events for the elderly. ${ }^{40}$ The reasons are related not only to the future size of the aging population or to their health status, but also to the lack of proper structure and different models of care. IMSS do not provide emergency transportation service from the patient's home to the clinic or hospital. Consequently, a simple technology such as the one evaluated in this paper could represent an alternative to respond more efficiently to the health needs of the elderly, providing patients with immediate clinical advice given by a trained nurse. Also, health services not based exclusively in clinics and hospitals should be implemented urgently. As an example, a national health policy of home visits has been incorporated in the health care systems of Spain, the United Kingdom, and New Zealand. ${ }^{41}$

Recently, reports of the effectiveness of nurse home visits to elderly people have been published ${ }^{42,43}$ without conclusive results. Stuck et $\mathrm{a}^{43}$ presented a systematic review and a meta-analysis that included 18 trials with a total of 13,447 individuals older than 75 years with the aim of evaluating the effect of home visits on functional status, admission to nursing homes, and mortality. The authors found that nurse home visits are effective if a multidimensional geriatric evaluation is integrated and elderly people with low risk and fewer deficits are included. All of the trials included were performed in populations in developed countries whose health systems have incorporated these care schemes. However, another meta-analysis found no positive effect on functionality, ${ }^{44}$ although the number of studies included was smaller. This lack of evidence was also reported by van Haastregt et al, ${ }^{45}$ who analyzed 15 trials based on community preventive home visits to old people.

In our study, the main outcome was the frailty phenotype proposed by Fried et $\mathrm{al}^{31}$ because it has a comprehensive perspective and can detect longitudinal effects, although the concept remains controversial. ${ }^{46}$ In that sense, the positive effect that we found for the $\mathrm{NV}+\mathrm{AB}$ group is consistent with some of the previous reports. ${ }^{43}$ We must accept that our results are difficult to compare due to the characteristics of the interventions that we evaluated. The evaluation of nonpharmacological interventions is complex. Content standardization of each visit and type and quality of interaction between nurses and patients cannot be analyzed, and it is not possible to know whether it was the assistive technology by itself or the combination with the nurses' work to provide closer and longitudinal care of the assigned patients was the determinant factor. It is noteworthy that the intervention group based only on home nursing visits had worse results, with patients presenting approximately $10 \%$ deterioration, as confirmed by regression analysis. These results cannot be explained by basal conditions. The main purpose of the nurse interaction with the patient would be lifestyle modification and a gain in quality of life measures; however, this group did not achieve this aim. ${ }^{47}$ Although all of the study nurses held bachelor's degrees, we must admit that nursing training profiles in Mexico are heterogeneous, and this could be one of the explanations for our results. However, the NV+AB group reported improvement in almost all components of frailty phenotype and even when these changes were slight, a visiting nurse combined with technology that produces a sense of security in the patient could diminish the level of risk. ${ }^{48}$ As van Haastregt et al ${ }^{45}$ proposed, substantial improvements must be made in the content and operative processes of these interventions if health care systems are to incorporate similar strategies for the care of the older people.

One of the interesting points of this work is the incorporation of alert buttons, which are a well-known assistive technology in other countries. This technology, combined with the triage protocols inserted in the mobile phone, was of great support in the processes of communication and decision making between the nurse and patient. Several technological devices may help to improve the care of the elderly, from electronic clinic files to telemedicine systems and prototypes to support daily life. ${ }^{49}$ Alert buttons are not a novel technology, but their utilization combined with the mobile phone may be a tool to make better clinical decisions to achieve closer patient care.

There are several limitations to the present study. The statistic power of the sample reached $78 \%$. However, some of the results would have been more evident if a higher number of participants had been included. It may be possible that this circumstance and some basal differences explain the positive effect observed in the control group. The randomization process was performed carefully. Although the staff in charge of the interventions could not be blinded to the allocations, this process was performed in the central module, as was 
the screening phase, and interviewers not involved with the intervention made the baseline and final evaluations. Furthermore, data input and analysis was performed by a research analyst not involved in the fieldwork team. Quality control was performed through supervision, although it was not possible to supervise each one of the visits. The content of the visits and the quality of the nursing recommendations was not analyzed, and the total number of visits was not included. It is likely that nurses working in the $\mathrm{NV}+\mathrm{AB}$ group would be more motivated to update their clinical knowledge because they had to resolve their patient's phone calls properly and promptly. This certainly could be a cause of the positive effect in this group.

Finally, we can say that it is necessary to complement this analysis with economic evaluations that provide more data to inform policy decision making. The quickly aging population in Mexico has left informal caregivers, mostly women, to assume much responsibility for the provision of household care activities through the life cycle, from early childhood care and family health care, to health and long-term care for the older population. ${ }^{50}$ Fundamental changes in social customs that brought about a constant increase in women's participation in formal education and employment outside the household are already posing a further challenge by reducing the supply of informal caregivers. Consequently, future research must be focused on evaluating long-term effects as well as how to increase the technical content of the interventions if we are to consider these strategies viable and valid for the care of the elderly in Mexico.

\section{Acknowledgments}

This project was supported by grants from CONACyT (México) SALUD-2007-C01-69834. The sponsor was the National Council of Science and Technology. Funds were obtained by a contest. CONACYT has no role in the design methods, subjects, or analysis other than to provide funds for the research.

\section{Author contributions}

CGP contributed to the concept, protocol, and original idea, coordinated the research, and reviewed the manuscript. JF participated in the original protocol, was in charge of the technological section, and reviewed the manuscript. LC participated in the technological section. FFM supported the conduct of the research and the data analysis. SSG, TJC, and CEB prepared the first draft of the paper and supervised the fieldwork. JMA participated in the original protocol and coordinated the fieldwork. MR coordinated the process of integrating the clinical data and participated in the integration of triage protocols to mobile phones.

\section{Disclosure}

The authors report no conflicts of interest in this work.

\section{References}

1. Palloni A, Pinto-Aguirre G, Pelaez M. Demographic and health conditions of ageing in Latin America and the Caribbean. Int J Epidemiol. 2002;31:762-771.

2. United States Census Bureau [webpage on the Internet]. International Programs [International database 2011]. US Census Bureau [updated August 15, 2012]. Available from: http://www.census.gov/ipc/www/ $\mathrm{idb} /$. Accessed May 10, 2011.

3. World Bank. Averting the Old Age Crisis: Policies to Protect the Old and Promote Growth. Washington DC: Oxford University Press; 1994.

4. Consejo Nacional de Población (CONAPO). Proyecciones de Población 2000-2050. [Population Projections 2000-2050]. México City, México: CONAPO; 2002. Spanish.

5. Tennstedt S, Crawford S, McKinlay J. Is family care on the decline? A longitudinal investigation of the substitution of formal long-term care services for informal care. Milbank Q. 1993;71:601-623.

6. Gordon M. Community care for the elderly: is it really better? Can Med Assoc J. 1993;148:393-396.

7. Abongomera L. Does Uganda need a policy for care of the elderly? World Health Forum. 1994;15:185-186.

8. Zúñiga Herrera E, García JE. El envejecimiento demográfico en México. Principales tendencias y características [The demographic aging in Mexico. Main trends and characteristics]. In: La Situación Demográfica de México 2008. México City, México: Consejo Nacional de Población; 2008:93-100. Spanish.

9. Hebert R, Robichaud L, Roy PM, et al. Efficacy of a nurse-led multidimensional preventive programme for older people at risk of functional decline. A randomized controlled trial. Age Ageing. 2001;30: 147-153.

10. Bouman A, van Rossum E, Ambergen T, et al. Effects of a home visiting program for older people with poor health status: a randomized, clinical trial in The Netherlands. J Am Geriatr Soc. 2008;56:397-404.

11. Huss A, Stuck AE, Rubenstein LS, et al. Multidimensional preventive home visit programs for community-dwelling older adults: a systematic review and meta-analysis of randomized controlled trials. J Gerontol A Biol Sci Med Sci. 2008;63A:298-307.

12. García-González JJ, García-Peña C, Franco-Marina F, Gutiérrez-Robledo LM. A frailty index to predict the mortality risk in a population of senior Mexican adults. BMC Geriatr. 2009;9:47.

13. Rockwood K, Mitnitski A, MacKnight C. Some mathematical models of frailty and their clinical implications. Rev Clin Gerontol. 2001;12: 109-117.

14. Mitnitski A, Song X, Skoog I, et al. Relative fitness and frailty of elderly men and women in developed countries and their relationship with mortality. JAm Geriatr Soc. 2005;53:2184-2189.

15. Lauritsen JM, Bruus M; EpiData Association. EpiTour - an Introduction to EpiData Entry. Odense, Denmark: The EpiData Association; 2001 [version 25th 2005]. Available from: http://www.epidata.dk/downloads/ epitour.pdf. Accessed May 10, 2011.

16. Charlson ME, Pompei P, Ales KL, et al. A new method of classifying prognostic comorbidity in longitudinal studies: development and validation. J Chronic Dis. 1987;40:373-383.

17. World Health Organization. International Statistical Classification of Diseases and Health Related Problems. Geneva: World Health Organization; 2004.

18. Reyes de Beaman S, Beaman P, García-Peña C, et al. Validation of a modified version of the Mini-Mental State Examination (MMSE) in Spanish. Neuropsychol Dev Cogn B Aging Neuropsychol Cogn. 2004;11:1-11. 
19. Franco-Marina F, García-González JJ, Wagner-Echeagaray F, et al. The Mini-mental State Examination revisited: Ceiling and floor effects after score adjustment for educational level in a Mexican ageing population. Int Psychogeriatr. 2010;22:72-81.

20. Folstein MF, Folstein SE, McHugh PR. "Mini-mental state". A practical method for grading the cognitive state of patients for the clinician. J Psychiatr Res. 1975;12(3):189-198.

21. Reyes-Ortega M, Soto-Hernández AL, Milla-Kegel JG, et al. Actualización de la escala de depresión del Centro de Estudios Epidemiológicos (CES-D). Estudio piloto en una muestra geriátrica Mexicana [Revision of the Center for Epidemiologic Studies Depression Scale (CES-D). Pilot study with a Mexican geriatric sample]. Salud Mental. 2003;26:59-68. Spanish.

22. Radloff LS. The CES-D scale: a self-report depression scale for research in the general population. Appl Psychol Measure. 1977;1:385-401.

23. Testa MA, Simonson DC. Current concepts: assessment of quality-of-life outcomes. N Engl J Med. 1996;334:835-840.

24. Ware JE Jr, Sherbourne CD. The MOS 36-item short-form health survey (SF-36). Conceptual framework and item selection. Med Care. 1992;30: 473-483.

25. Zuñiga MA, Carrillo-Jimenez GT, Fox PJ, et al. Evaluación del estado de salud con la Encuesta SF-36: resultados preliminares [Health status evaluation with the SF-36 survey: preliminary results in Mexico]. Salud Publica Mex. 1999;41:110-118. Spanish.

26. Hays RD, Sherbourne CD. RAND 36-Item Health Survey 1.0 Scoring Manual. Santa Monica, CA: The RAND Corporation; 1992.

27. Katz S, Ford AB, Moskowitz RW, et al. Studies of illness in the aged. The index of ADL: a standardized measure of biological and psychosocial function. JAMA. 1963;185:914-919.

28. Lawton MP, Brody EM. Assessment of older people: self-maintaining and instrumental activities of daily living. Gerontologist. 1969;9: 179-186

29. World Health Organization. Physical Status: The Use and Interpretation of Anthropometric Physical Status. Report of a WHO Expert Committee. Geneva: World Health Organization; 1995.

30. Rubenstein LZ, Harker JO, Salva A, Guigoz Y, Vellas B. Screening for undernutrition in geriatric practice: Developing the Short-Form Mini Nutritional Assessment (MNA-SF). J Gerontol. 2001;56A M366-M377.

31. Fried LP, Tangen CM, Waltson J, et al. Frailty in older adults: evidence for a phenotype. J Gerontol Med Sci. 2001;56A:M146-M156.

32. Roper WL, Baker EL Jr, Dyal WW, Nicola RM. Strengthening the public health system. Public Health Rep. 1992;107:609-615.

33. Hallal P, Gómez LF, Parra D, et al. Lecciones aprendidas después de 10 Años del uso de IPAQ en Brasil y Colombia [Lessons learned after 10 years of IPAQ use in Brazil and Colombia]. J Phys Activity Health. 2010;7(Supp1 2):S259-S264. Spanish.

34. Castro LA, Favela J, García-Peña C. Naturalistic enactment to stimulate user experience for the evaluation of a mobile elderly care application. In: Proceedings of the 13th International Conference on Human Computer Interaction with Mobile Devices and Services (MobileHCI '11). New York, NY: ACM; 2011:371-380
35. Briggs JK. Telephone Triage Protocols for Nurses. Philadelphia, PA: Lippincott Williams \& Wilkins; 2002.

36. StataCorp. Stata Statistical Software: Release 12. College Station, TX: StataCorp LP; 2011.

37. Lehtonen R, Pahkinen E. Practical Methods for Design and Analysis of Complex Surveys (Statistics in Practice), 2nd ed. West Sussex, UK: John Wiley \& Sons Ltd; 2004.

38. Hosmer DW, Lemeshow S. Applied Logistic Regression, 2nd ed. New York, NY: John Wiley \& Sons; 2000.

39. Organization of Economic Cooperation and Development. Health at a Glance 2011: OECD Indicators. OECD Publishing; 2011. Available from: http://www.oecd-ilibrary.org/social-issues-migration-health/ health-at-a-glance-2011_health_glance-2011-en. Accessed May 10, 2011.

40. Gonzalez-Gonzalez C, Sánchez-García S, Juarez-Cedillo T, et al. Health care utilization in the elderly Mexican population: expenditures and determinants. BMC Public Health. 2011;11:192.

41. Byles JE. A thorough going over: evidence for health assessments for older people. Aust N Z J Public Health. 2000;24:117-123.

42. Fleischer S, Roling G, Beutner K, et al. Growing old at home a randomized controlled trial to investigate the effectiveness and cost-effectiveness of preventive home visits to reduce nursing home admissions: study protocol. BMC Public Health. 2008;8:185.

43. Stuck A, Egger M, Hammer A, et al. Home visits to prevent nursing home admission and functional decline in elderly people. Systematic Review and meta-regression analysis. JAMA. 2002;287:1022-1028.

44. Elkan R, Kendrick D, Dewey M, et al. Effectiveness of home-based support for older people. BMJ. 2001;323:719-724.

45. van Haastregt J, Diedericks J, Rossum E, et al. Effects of preventive home visits to elderly people living in the community: systematic review. BMJ. 2000;320:754-758.

46. Avila-Funes JA, Aguilar-Navarro S, Melano-Carranza E. La fragilidad, concepto enigmático y controvertido de la geriatría: la visión biológica [Frailty, an enigmatic and controversial concept in geriatrics: the biological perspective]. Gaceta Médica México. 2008;144(3):255-262. Spanish.

47. Steffen F, Berg A, Zimmermann M, et al. Nurse-patient interaction and communication: A systematic literature review. J Public Health. 2009; 17:339-353.

48. Espinoza S, Walston JD. Frailty in older adults: insights and interventions. Cleveland Clin J Med. 2005;72(12):1105-1112.

49. Piette J, Lun KC, Moura L, et al. Impacts of e-health on the outcomes of care in low- and middle-income countries: where do we go from here? Bull World Health Org. 2012;90:365-372.

50. Horvath L, Mayer S. Caring for informal caregivers: policy approaches to the provision of direct support services. Innovation: The European Journal of Social Science Research. 2010;23(3):263-277.
Clinical Interventions in Aging

\section{Publish your work in this journal}

Clinical Interventions in Aging is an international, peer-reviewed journal focusing on evidence-based reports on the value or lack thereof of treatments intended to prevent or delay the onset of maladaptive correlates of aging in human beings. This journal is indexed on PubMed Central, MedLine, the American Chemical Society's 'Chemical Abstracts

\section{Dovepress}

Service' (CAS), Scopus and the Elsevier Bibliographic databases. The manuscript management system is completely online and includes a very quick and fair peer-review system, which is all easy to use. Visit http://www.dovepress.com/testimonials.php to read real quotes from published authors. 